\title{
Design of Rotary Transformer Axis-Angle Digital System Based on AD2S83
}

\author{
Zhou Guo-jun \\ Department of Basics \\ Dalian Naval Academy \\ Dalian, China \\ zhou.guojun@163.com
}

\author{
Cheng Jin, Zhou Bo \\ Department of Basics \\ Dalian Naval Academy \\ Dalian, China \\ judyever@sina.com
}

\begin{abstract}
This paper introduces the design of rotary axis-angle digital system, which is composed of integrated angle-to-digital converting circuit AD2S83, single chip computer and huge capacity memory. This system converts the analog signals and reference signals from rotary transformer to digital information that denotes angle position and angular speed. It can also handle, memorize, display and transmit the converting result and has of practical value in research and development of monitor and control system of the angular position.
\end{abstract}

Keywords-rotary transformer; axis-angle; conversion; micro computer unit (MCU); $A D 2 S 83$

The rotary transformer has been widely used in various control systems of aviation, space, navigation and etc, such as radar direction, satellite navigation, fire control system, target detection system, servo system, machine tool control system, and etc, because it is reliable and durable and can output high-precision axis-angle position information. Because of the output of the rotary transformer contains the analog signals of the axis-angle information, recently, various kinds of axis-angle/digital transformer have continuously appeared in order to realize the requirement of the complex digitalized control. AD2S83 made of BIMOS II techniques developed by AD Company is a MCU rotary transformer/digital conversion (RDC) by combining the advanced CMOS logic circuit with high-precision bipolar circuit. This powerful, high-reliable chip, together with instruments like a high-performance MCU can realize the digitalized conversion, storage, transmission and handle of the axis-angle information like the absolute position, angular speed, displacement direction, and etc, and is widely used in a variety of digital measure-control systems. The writer designs the practical rotary transformer axis-angle digital system with the use of AD2S38 combining the high-performance MCU and other instruments[1].

\section{AD2S83 PRINCIPLES AND CHARATERISTICS}

\section{A. Principle of Rotary Transformer Measuring Angle}

The rotary transformer is a measuring mechanical axis-angle control motor, as shown in Figure-1, by adding the alternating-current exciting voltage to its stator winding $\mathrm{S}_{1}-\mathrm{S} 2$, and the expression is[2] [3]:

$$
u_{f}=U_{f m} \sin \omega t
$$

Then its rotor winding output and the axis-angle is a strict $\sin /$ cosine relation, and the expression is :

$$
\begin{aligned}
& u_{s}=U_{m} \sin \theta \sin \omega t \\
& u_{c}=U_{m} \cos \theta \sin \omega t
\end{aligned}
$$

In the expression, $\theta$ is the included angle between the rotor cosine win-ding the axis line $\mathrm{R}_{1}-\mathrm{R}_{2}, \mathrm{U}_{\mathrm{m}}$ is the amplitude of the cosine winding the induced alternating current w-hen $\theta=0$, the numerical values of $\mathrm{u}_{\mathrm{s}}$ and $\mathrm{u}_{\mathrm{c}}$ reflect the size of rotary angle.

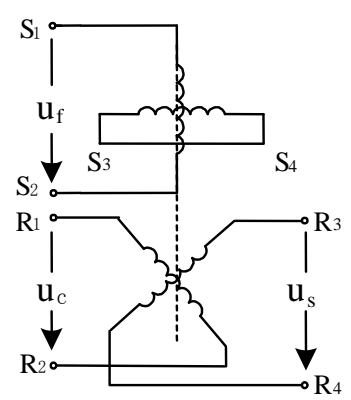

Figure 1. Principle Picture of the Rotary

\section{B. Principle of AD2S83 Conversion}

AD2S83 is a tracking convertor by using the digital phase lock technology, and its working principle picture is shown in Figure-2.

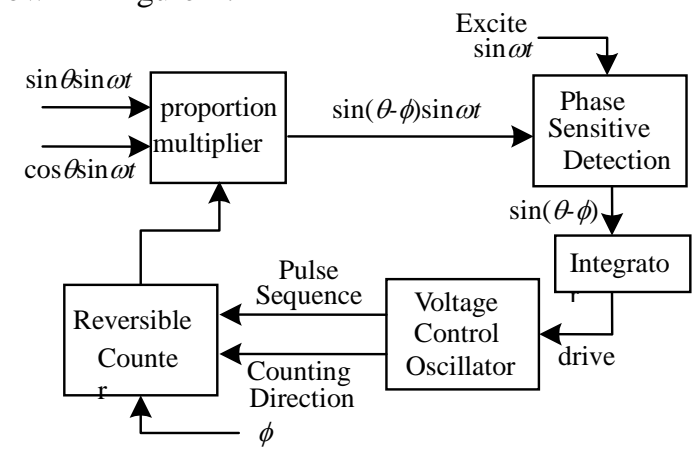

Figure 2. AD2S83 working principles

In the Figure 2, $\theta$ stands for the axis-angle, $\phi$ stands for the digital state angle of the reversible counter, $\phi$ tracks the axis-angle $\theta$ all the time, the difference cosine between $\theta$ and $\phi$ is zero, and then the digital value of the axial angle can be decided[4] [5] [6]. 


\section{Characteristics of AD2S83 Chip}

- Allow the customer to select the suitable resolution by themselves. AD2S83 offers 10 bit, 12 bit, 14 bit, or 16 bit resolution, and the customer can select the different resolutions via the different connections of the outer instruments based on their requirements.

- Use the binary code paralleled by the pin output via three state output to signify the position information, then it is easy to interface the control chip like the MCU or DSP, etc.

- Adopt the proportion-track conversion pattern to keep it to output the position data continuously while avoiding conversion delay and keeping its stronger anti-jamming capability and long-distance transmission capability.

- Allow the customer to select the outer resistance and capacitance elements to change the dynamic performance of the conversion, such as the band width, the biggest tracking rate, etc.

- Have a very high tracking speed, the biggest tracking speed of 10 bit resolution is $1040 \mathrm{r} / \mathrm{s}$.

- Provide high precision speed signal output. AD2S83 can provide the analog signal in direct proportion to the rotary speed, and its typical degree of linearity reaches $\pm 1 \%$, backlash less than $\pm 0.3 \%$, which can replace the measuring speed generator[7].

\section{DESIGN OF THE SYSTEM HARDWARE}

The rotary transformer axis-angle digital system is made up of AD2S83 and its outer circuit, ADuC812 MCU, memory, clock, LED display module, etc, and the pane picture of its components is shown in Figure 3.

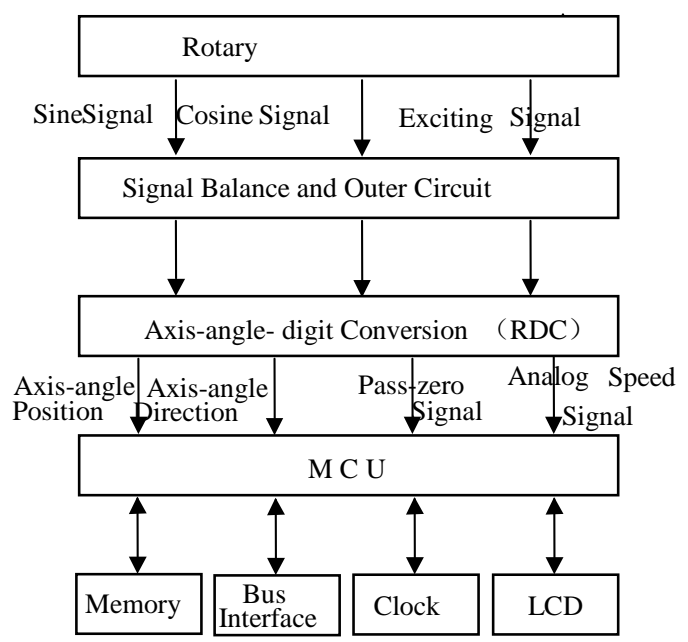

Figure 3. The rotary transformer axis-angle digital system diagram

In accordance with the two phase analog signal cont-ained axis-angle information of the specific rotary tran-sformer and the amplitude of the exciting signal, the balancing resistance of the AD2S83 input end can be adjusted to match the amplitude of input signal. The axis-angle/digital conversion circuit outputs the signal of the axis-angle position, the rotary direction, and etc in the form of digital value, and the axis-angle speed signal in the form of the analog value. The MCU collects the output of the axis-angle/digital conversion circuit, and calculates, memorizes and processes according to the requirements; the LED display module displays real time various information of the axis-angle according to the requirements, including the malfunctioned state, and the simple keyword board can be used to send the control order of memory, transmission, and etc via Chinese character interface. The MCU can communicate with the upper computer through RS485 bus interface, receive the order of working pattern and etc, and collect, submit, handle or memorize data[8].

\section{A. Outer circuit of $A D 2 S 83$}

Figure 4 is the typical circuit by using AD2S83 to realize the axis-angle/digital conversion. The digital value of each element in the Figure can be drawn under the condition that the resolution is $10 \mathrm{bit}$, the reference signal frequency is $5 \mathrm{kHz}$ and the biggest tracking rate is $260 \mathrm{rps}$. Under different applicable conditions and requirements, the criteria of each element can be calculated according to the customer manual.

AD2S83 outputs the speed signal in the form of analog voltage (42 pin), and the biggest output amplitude is $\pm 8 \mathrm{~V}$. The 25 pin to 10 pin of the chip corresponding to 16 bit axis-angle position outputs DB16-DB1, which can select 8 bit or 16 bit to output by BYTE SELECT. When BUSY is low level, its output data is valid. DIRECTION indicates the changing direction the axis-angle. When the output data changes from full " 1 " to full " 0 " or from full " 0 " to full "1", RIPPLE CLOCK end outputs a rising jump. From the above output signals, the axis-angle position, rotary speed and direction information can be drawn and accelerating speed and revolution circles can be gained after further handling.

The 27 pin of AD2S83 controls the state of the data output DB16-DB1, and the content of internal output data latch appears on the DB16-DB1 when in low level, and DB16-DB1 bit shows high impedance when in high level; 30 pin controls the internal data latch, and forbids to refresh the internal data latch when in low level. The two control signals can make the AD2S83 easily interface the MCU.

\section{B. MCU Interface Circuit}

Considering the analog speed signal output by AD2S83 is converted into $\mathrm{A} / \mathrm{D}$ and large capacity of data needs memorizing, it is appropriate to select the ADuC812 MCU made by American Analog Instruments Company as the system controller. ADuC812 $\mathrm{MCU}$ is a complete high-performance data collecting system chip, which includes 8 bus 12 bit ADC module, 2 bus 12 bit DAC module, $8052 \mathrm{MCU}$ core, $8 \mathrm{~KB}$ flash/electricity erasable program memory, 640 byte flash/electricity erasable program memory, watchdog timer, source monitor, I2C compatible two lines and SPI series interface, standard UART series interface and a flexible electricity management project. Due to ADuC812's rich chip-in resources, I/O interface, and more than $16 \mathrm{M}$ byte outer data memory addressing ability, it is fully functional, universal, wide range for working temperature, fit for the bad environment and easy to be used in collecting and measuring data and the control system. 
The relation of interface connection between $\mathrm{ADuC} 812$ $\mathrm{MCU}$ and AD2S83 instrument is shown in Figure 5. signal amplitude output by $\mathrm{AD} 2 \mathrm{~S} 83$ can reach $\pm 8 \mathrm{~V}$, it needs to adjust the amplitude of the analog speed signal

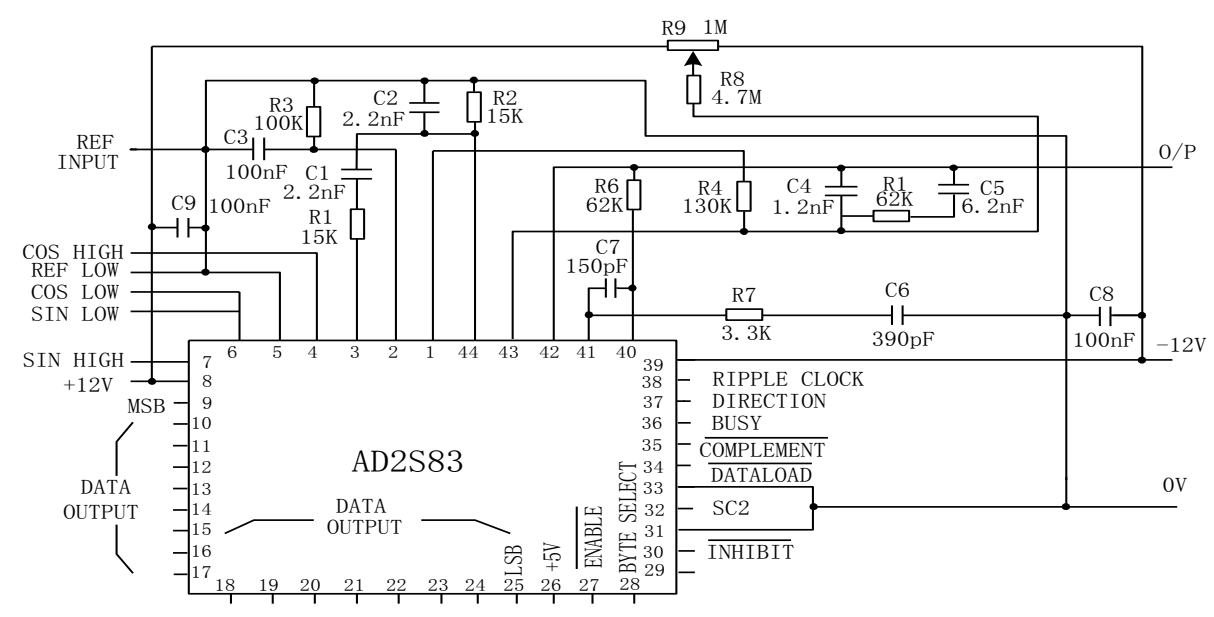

Figure 4. Typical outer circuit of AD2S83 axis-angle/ digital conversion

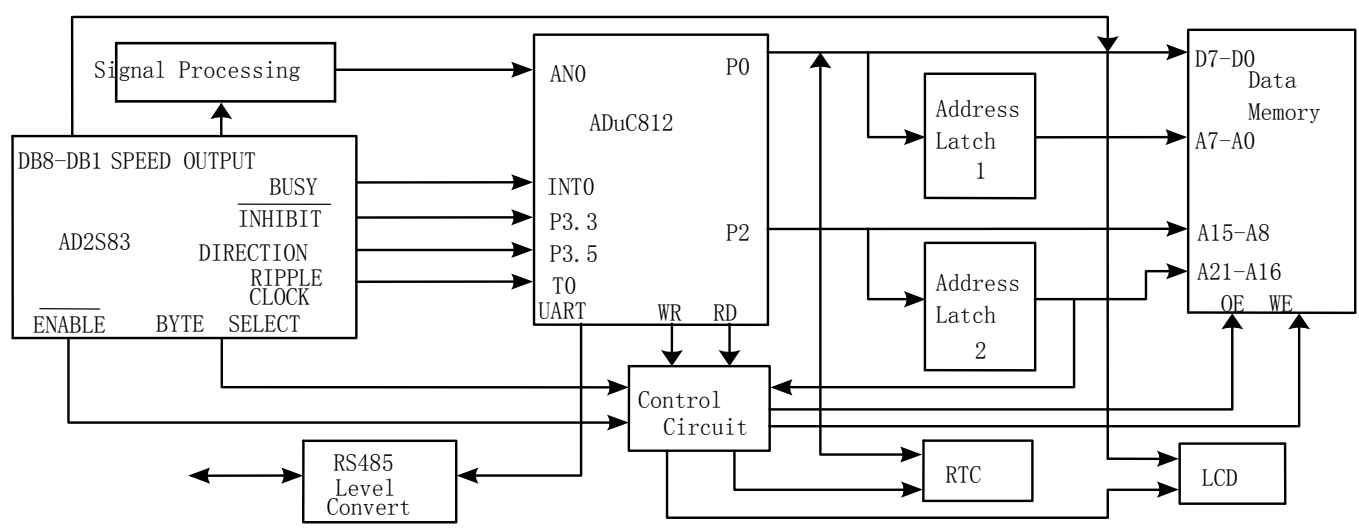

Figure 5. Block representation of MCU interface

The BUSY signal of AD2S83 is connected with the input end of MCU external interrupt 0 . When the axis-angle position changes, AD2S83 will track and convert to keep BUSY in high level. After the conversion, it becomes low level and the indicating data is valid, and the fall edge of BUSY makes the MCU enter the interrupt service process to read the axis-angle position data. While the reading can be divided into two times, high 8 bit can be read when placing BYTE SELECT into 1; low 8 bit when placing BYTE SELECT into 0 . When reading data, it is forbidden to refresh the internal data latch by placing P3.3 into low level, and this can ensure the reading data to be correct[9].

By making MCU's timing/counter 0 work for the counting method, the past-zero times of the axis-angle position can be recorded, then the revolution circle number of the axis can be gained or the MCU can be informed by the interrupt method after the revolution circle number reaches the given value.

Since the input signal range of ADuC812 MCU ADC module is limited from $2 \mathrm{~V}$ to $2.5 \mathrm{~V}$, and the analog speed and move the electrical level by using the signal to adjust the circuit. By setting AduC812 MCU specific function register $\mathrm{ADCCON} 1$ and $\mathrm{ADCCON} 2$, it can make the $\mathrm{ADC}$ module of the AduC812 work according to the needed converting time and converting method, and can inquire the work state of ADC by ADCCON3. When the ADC module works in the form of DMA, the converting rate can reach $200 \mathrm{KHz}$.

NVRAM with the Capacity $4 \mathrm{Mx} 8$ can be used as the data memory, and the type is HK12A5. While it doesn't need power failure protection, the corresponding SRAM can be used.

\section{DESIGN OF THE SYSTEM SOFTWARE}

The system software adopts the MCU C language program to make the process structure clear, transplantable and well-improved maintainable. The software system employs the interrupt drive, modularized structure, and comprises main process, interrupt service process and task process module.

The task process module contains series communication, control order processing, ADC module 
arrangement, analog signal collection, digital signal collection, data processing, DMA regional initialization, LED display, etc, and each module corresponds to one task. The state of each task reflects the main process by the state symbol. The main process decides the task state by constantly inquiring the task state symbol transfers the task process module to perform the corresponding task and makes the corresponding handle[10].

\section{CONCLUSION}

The rotary transformer axis-angle digital system consisted of single axis-angle/digital converting integrated circuit AD2S83, ADuC812 MCU and large capacity memory and etc can collect, process, memorize, display or transmit various axis-angle information, work independently or link a net, and have a practical value in the research, development and state-monitor of the rotary transformer system. If the measuring angle element is a self-handle angle machine, then the 3-phase signal of the self-handle angle machine can be converted into the $\sin / \mathrm{cos}$ signal in the format of the rotary transformer with the use of the SCOTT electron transformer circuit, and, which is connected to this system, that is a self-handle angle machine axial-angle digit system.

\section{REFERENCES}

[1] Ye Xiaoxue and Wang Xinmin, "Design of multi-pole resolver angle data acquisition card," Foreign Electronic Measurement Technology, vol. 2,pp.68-71,Feb.2010

[2] Han Jie, Wang Hong-cheng, "Kind of rotor position and speed test unit based on AD2S83," Electrotechnics Electric , vol. 4,pp.26-28,Apr.2011

[3] Zhuang Wen-xu,MA Da-wei, “A position sensor module Design for Dual-channel resolver based on AD2S83," Small \& Special Electrical Machines, vol.40(5),pp.27-29,May.2012

[4] WANG Anm in, CUIWei,NIU Chuanmeng,"Angle Measurement System of Round Inductosyn Based on AT89C52 and AD2S 83 , "Instrument Technique and Sensor,9,pp.55-57,Sep.2009

[5] LIANG Hong,ZHOU Ai-jun, "Design of Synchronizer Axis-angle Data Acquisition Recorder", Instrument Technique and Sensor, vol. 7,pp.7-9,Jul.2005

[6] DOU Jin sheng, LI Ming, TANG Tian hao, "A new simulative compass and its control," vol. 5,pp.63-65,May.2006

[7] GUO Jia, FAN Zhen-nan, "Intelligent Node Design to Measure Angle Displacement Based on Inductosyn,Science Technology and Engineering,Vol. 11 (27 ),pp.6637-6642,Sep.2011

[8] Jin Jingqiang,Wu Fuchun. "Design and Implementation of signal interface circuit of rotary transformer,"Computer Development \& Applications, vol. 5,pp.54-56,May.2007

[9] XU Da-lin,CHEN Jian-hua,"Technology and development of synchro /resolver to digital conversion,"Measurement \& Control Technology, Vol. 24(10),pp:1-5,Oct.2005

[10] LI Li yu,ZHANG Shuang-xi, "Application of resolver position digital decoding to tank gun control system,"Fire Control \& Command Control,Vol. 36(11),pp:147-151,Nov.2011 